\title{
Compositional Transient Stability Analysis of Power Systems via the Computation of Reachable Sets
}

\author{
Ahmed El-Guindy, Yu Christine Chen, and Matthias Althoff
}

\begin{abstract}
The main challenge associated with the analysis of power systems via the computation of reachable sets is improving the algorithmic efficiency to scale towards industrially relevant problem sizes. In this paper, we present a compositional algorithm that can drastically reduce the computational effort required to assess the dynamical response of power systems during transients using reachability analysis. The main reason for the algorithmic efficiency is that we reformulate the transmission network into a set of subsystems, each consisting of a synchronous generator connected to a generator bus, whose algebraic constraints are unknown-but-bounded within some confidence intervals. This makes it possible to parallelize the computation of reachable sets for transient stability analysis and, more importantly, preserve the interaction and correlation between different machines connected to the grid. The applicability of the proposed compositional algorithm is illustrated on several benchmark examples and compared to other algorithms that compute the reachable set without employing any compositional techniques.
\end{abstract}

Index Terms - Transient stability analysis, dynamic security assessment, power systems, reachability analysis

\section{INTRODUCTION}

Recently, reachability analysis has emerged as an alternative and promising technique for the analysis of power systems. A general literature review about reachability analysis is found in [1]-[3]. Basically, reachability analysis makes it possible to compute bounds of all system trajectories, starting from a set of unknown initial states, while simultaneously considering the influence of parametric and input uncertainties. These uncertainties are typically associated with various fault scenarios, and/or renewable resources that are continuously integrated into the grid.

The applicability of reachability analysis in power systems has been reported in a wide range of applications, such as, cyber-security [4], [5], assessment of conventional power plants [6] and wind turbines [7], load flow and static performance [8], [9], reachability-based control synthesis [10], [11], estimation of stability regions [12], [13], and transient stability analysis [14], the main focus of this paper. Transient stability analysis dates back to the 1920s [15] and is widely recognized technically and historically among theorists and practitioners alike as the most problematic issue when considering the dynamic security assessment of power systems [16]. Simply put, transient stability refers to the ability of synchronous generators to remain in synchronism

A. El-Guindy and M. Althoff are with the Institute of Robotics and Embedded Systems, Technische Universität München, Garching, Germany. E-mail: \{ahmed.elguindy, althoff\}@tum.de

Y. C. Chen is with the Department of Electrical and Computer Engineering at the University of British Columbia, Vancouver, Canada. E-mail: chendece.ubc.edu with the frequency of the utility grid following the event of a large disturbance in the transmission network [17], [18].

Early contributions applying reachability analysis for this class of problems in power systems were reported in [12], [19], [20]. These algorithms rely on an Eulerian scheme that employs level-set methods (LSMs) to compute backward reachable sets starting from a target set. This is achieved via the formulation of a Hamilton-Jacobi-Isaacs (HJI) partial differential equations (PDEs), where it is proven that the viscosity solution of the time-dependent HJI PDEs provides an implicit surface representation of the continuous backward reachable set [21]. This makes it possible to estimate a region of attraction from which one can identify the initial states of the post-fault scenario that converge back to the equilibrium. Along the same lines, the contributions presented in [22], [23] formulate power systems as a hybrid automatan to compute forward reachable sets using techniques based on LSMs for hybrid systems.

The main drawback of this class of techniques, however, is that the computational requirements grow rapidly with the system dimension due to the fact that no analytical solution exists for the set of PDEs. Thus, the state space has to be continuously discretized, resulting in an exponential complexity with respect to the number of continuous state variables. This limited the applicability of LSMs to the Single-Machine Infinite-Bus (SMIB) and the Double-Machine Infinite Bus (DMIB) benchmark problems, in which only a maximum of five state variables have been reported. Another limitation of the LSMs is that it only provides an accurate approximation of the reachable set, rather than a rigorous enclosure of it; hence, it does not hold as a formal technique [21].

The alternative class of methods for reachability computation is based on Lagrangian techniques, which compute reachable sets similar to numerical integration methods. This is achieved by propagating the set of reachable states instead of only computing the solution for a single point in time. Although there exists a large variety of well-developed methods that consider nonlinear systems of ordinary differential equations (ODEs), such as abstraction via local linearization [24], [25] or Taylor models [26], there is, however, little work regarding an efficient algorithmic procedure for computation of reachable sets with rigourous bounds for power systems described via the standard formalization using differential algebraic equations (DAEs). One reason is that an extension of reachability algorithms based on Lagrangian schemes for ODEs to handle DAEs is necessary. This task, however, is not straightforward since the class of DAE systems differs in both theoretical and numerical properties [27]. 
In our previous work, we have developed a numerical procedure to compute reachable sets for the class of power systems modelled via DAEs. Although the proposed algorithm has a polynomial complexity $\mathcal{O}\left(n^{5}\right)$, with $n$ corresponding to the number of state variables, the computational requirements were enormous for transient stability analysis of the IEEE 30-bus benchmark problem [14]. The main contribution of this paper is a compositional algorithm for transient stability of power systems modelled by standard DAEs, whose computational and associated memory requirements grow moderately with the system dimension, in comparison to our previous contributions. The proposed methodology makes it possible to verify each subsystem in the grid separately, while preserving the interaction and correlation between synchronous machines during fault scenarios. This allows one to parallelize the computation of reachable sets, which drastically reduces the computational efforts.

\section{PReliminaries}

We denote by $\mathbb{N}^{n}, \mathbb{R}^{n}$ the set of natural and real numbers with dimension $n$. For two sets $\mathcal{X}, \mathcal{Y} \subset \mathbb{R}^{n}$, the operators $\oplus, \times$ return the Minkowski sum and Cartesian product, respectively. The convex hull enclosure of two sets is denoted by $\mathbf{C H}(\cdot)$ whereas the interval enclosure is $\mathbf{I H}(\cdot)$. For two vectors $\underline{a}, \bar{a} \in \mathbb{R}^{n}$ with $\underline{a}<\bar{a}$, a closed multidimensional interval is $\mathcal{I}=[\underline{a}, \bar{a}]$.

\section{A. Basic Operations on Zonotopes}

In this work, we use zonotopes to represent reachable sets. A zonotope is a centrally-symmetric convex polytope, expressed using the G-representation as follows

$$
\mathcal{Z}=(c, G)_{\mathcal{Z}}=\left\{x \in \mathbb{R}^{n_{\mathcal{Z}}}: x=c \oplus G \beta, \beta_{i} \in[-1,1]\right\},
$$

where $c \in \mathbb{R}^{n_{\mathcal{Z}}}$ is the zonotope center and $G \in \mathbb{R}^{n_{\mathcal{Z}} \times p}$ denotes the generator matrix, with $\beta \in \mathbb{R}^{p}$.

Now we present the basic operations performed on zonotopes, in order to compute reachable sets as we describe later in Section III-B. These operations are the Minkowski sum, linear transformation, convex and box enclosure, and the Cartesian product. The aforementioned operations are illustrated in Fig. 1.

We introduce the square matrix $M \in \mathbb{R}^{n_{\mathcal{Z}} \times n_{\mathcal{Z}}}$ in addition to two zonotopic sets of equal dimensions $\mathcal{Z}_{1}:=\left(c_{1}, G_{1}\right)_{\mathcal{Z}}$ and $\mathcal{Z}_{2}:=\left(c_{2}, G_{2}\right)_{\mathcal{Z}}$. The Minkowski sum of zonotopes is defined by

$$
\begin{aligned}
\mathcal{Z}_{3}: & =\mathcal{Z}_{1} \oplus \mathcal{Z}_{2} \subset \mathbb{R}^{n_{\mathcal{Z}}} \\
& =(\underbrace{\left[c_{1}+c_{2}\right]}_{=: c_{3}}, \underbrace{\left[g_{1}^{(1)}, \ldots, g_{1}^{\left(p_{1}\right)}, g_{2}^{(1)}, \ldots, g_{2}^{\left(p_{2}\right)}\right]}_{=: G_{3}})_{\mathcal{Z}},
\end{aligned}
$$

and the linear transformation of the resulting zonotope $\mathcal{Z}_{3}=\left(c_{3}, G_{3}\right)_{\mathcal{Z}}$ by the matrix $M$ is

$$
M \cdot \mathcal{Z}_{3}:=\left(M \cdot c_{3}, M \cdot\left[g_{3}^{(1)}, \ldots, g_{3}^{\left(p_{1}+p_{2}\right)}\right]\right) .
$$

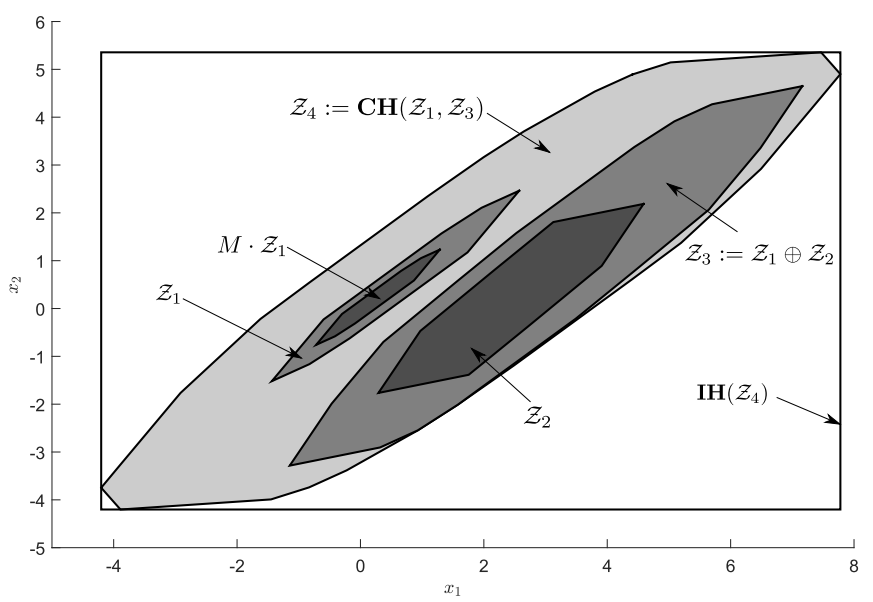

Fig. 1. Basic operations performed on Zonotopes throughout the paper, with $\mathcal{Z}_{1}$ and $\mathcal{Z}_{2}$ as two zonotopic sets of equal dimensions. The set $\mathcal{Z}_{3}$ results from the Minkowski sum and $\mathcal{Z}_{4}$ from the convex enclosure. The black solid box denotes the interval enclosure of the zonotope $\mathcal{Z}_{4}$.

Note that both operations are closed and result in another set described by a zonotope as well, which is one of the many advantages of using zonotopes for reachability computations.

The over-approximation of a zonotope using an interval hull is denoted by the operator $\mathbf{I H}(\cdot)$, which returns an axis-aligned bounding box expressed by

$$
\begin{aligned}
& \mathbf{I H}\left(\mathcal{Z}_{3}\right):=[\underline{\eta}, \bar{\eta}] \\
& \text { with } \underline{\eta}=c_{3}-\sum_{i=1}^{p_{1}+p_{2}}\left|g_{3}^{(i)}\right|, \quad \bar{\eta}=c_{3}+\sum_{i=1}^{p_{1}+p_{2}}\left|g_{3}^{(i)}\right|,
\end{aligned}
$$

and the convex hull operator $\mathbf{C H}(\cdot)$ required to enclose two zonotopes by another zonotope is

$$
\begin{aligned}
\mathcal{Z}_{4} & :=\mathbf{C H}\left(\mathcal{Z}_{1}, \mathcal{Z}_{3}\right) \\
& \subseteq \frac{1}{2}\left(\left[c_{1}+c_{3}\right],\left[G_{1}+G_{3}, G_{1}-G_{3}\right]\right)_{\mathcal{Z}} .
\end{aligned}
$$

Note that this operation is computed in an overapproximative manner, since the convex enclosure of two zonotopes is generally not a zonotope [24]. Finally, the Cartesian product of two zonotopes is

$$
\mathcal{Z}_{1} \times \mathcal{Z}_{2}=\left(\left[\begin{array}{l}
c_{1} \\
c_{2}
\end{array}\right], \quad\left[\begin{array}{cc}
G_{1} & \mathbf{0} \\
\mathbf{0} & G_{2}
\end{array}\right]\right)_{\mathcal{Z}}
$$

where $\mathbf{0}$ is a matrix of zeros with proper dimensions.

Remark 1. The reachability algorithm we will present later in Section III-B is in principle applicable for all kinds of set representation, e.g. polytopes [28], ellipsoids [29], and support functions [2]. However, we use zonotopes in particular due to their computational advantages. For example, the interval hull of zonotopes can be efficiently computed, which is advantageous for the computation of the Lagrangian remainder, to bound the set of the linearization errors. Moreover, zonotopes offer an excellent compromise between accuracy and efficiency compared to the aforementioned set representations, since the complexity of representing polytopes grows exponentially with the system dimension. Ellipsoids, on the other hand, are not closed under Minkowski sum. 


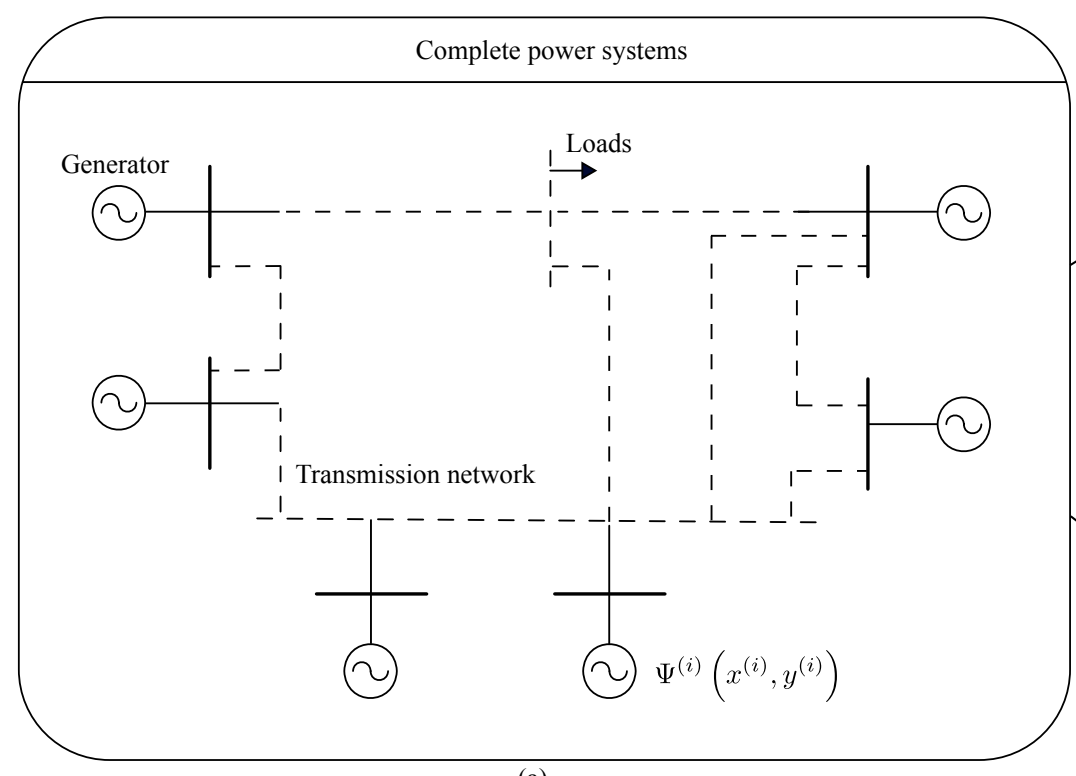

(a)

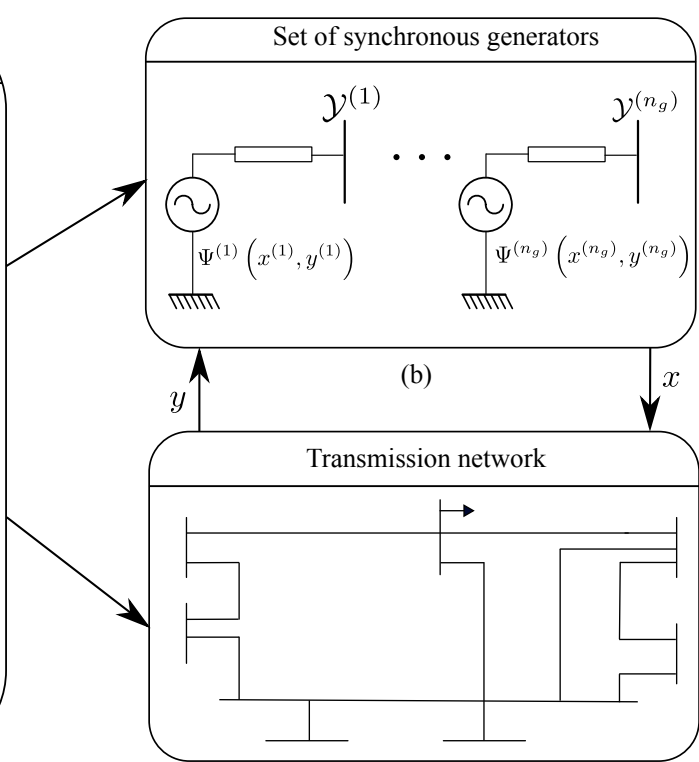

(c)

Fig. 2. Illustration of the compositional approach. In (a) the complete power system is modelled using the set of DAEs as described in (7). This system is reformulated into the compositional model (b) proposed as in (10), and the transmission network (c), which solves a set of nonlinear algebraic equations. The interaction between synchronous machines is preserved since the algebraic constraints corresponding to each generator's bus are not kept constant, but rather are known to vary within some confidence intervals. These intervals vary depending on the evolution of the synchronous machine state variables with respect to time and fault scenario.

\section{B. Problem Formulation and Objective}

We consider power systems described by standard models formalized by a set of time-invariant, semi-explicit, nonlinear, index-1 DAEs [18], [30]

$$
\begin{aligned}
\dot{x}(t) & =f(x(t), y(t)), \\
0 & =g(x(t), y(t)),
\end{aligned}
$$

with $f: \mathbb{R}^{n+m} \mapsto \mathbb{R}^{n}$ and $g: \mathbb{R}^{n+m} \mapsto \mathbb{R}^{m}$. Here, the vector $x \in \mathbb{R}^{n}$ includes the dynamic states variables of the synchronous machines, and the states constrained by the algebraic variables are included in the vector $y \in \mathbb{R}^{m}$. The time dependency is often omitted for simplicity of notation.

The objective of this paper is to assess the stability of the power system during transients by computing the reachable set of the dynamic states variables of (7) over a time horizon $t \in\left[0, t_{f}\right]$ starting from a set of consistent initial states $\mathcal{R}(0)$ and a set of possible inputs $\mathcal{Y}$. Due to the fact that power systems contains hundreds of states variables, the computational efforts associated with the reachable set computation can be drastically reduced using compositional techniques. That is, each subsystem in (7) can be verified separately by computing its reachable set described by

$$
\begin{aligned}
& \mathcal{R}^{(i)}\left(\left[0, t_{f}\right]\right):= \\
& \left\{x^{(i)}(t) \in \mathbb{R}^{n_{x}}: x^{(i)}(t)=\int_{0}^{t} \Psi^{(i)}\left(x^{(i)}(\tau), y^{(i)}(\tau)\right) d \tau,\right. \\
& \left.\quad x^{(i)}(0) \in \mathcal{R}^{(i)}(0), y^{(i)}(t) \in \mathcal{Y}^{(i)}, t \in\left[0, t_{f}\right]\right\},
\end{aligned}
$$

where the superscript $i$ corresponds to the $i$-th subsystem whose dynamics are modelled by the nonlinear function $\Psi(\cdot)$. The reachable set of the complete power system (7) is obtained by aggregating the reachable set of the $i \in\left\{1, \ldots, n_{g}\right\}$ subsystems, that is

$$
\mathcal{R}\left(\left[0, t_{f}\right]\right):=\mathcal{R}^{(1)}\left(\left[0, t_{f}\right]\right) \times \cdots \times \mathcal{R}^{\left(n_{g}\right)}\left(\left[0, t_{f}\right]\right) .
$$

Here it is worth noting that (8) corresponds to the exact reachable set, which is difficult or even impossible to compute [31]. Thus, an over-approximation including all behaviours of the nonlinear system is evaluated as tightly as possible.

\section{COMPositional Algorithm}

\section{A. Partitioning of Power Systems}

We assume that the system in (7) contains $n_{g}$ buses associated with the buses connected to a synchronous generator with the dynamic variables $x^{(i)} \in \mathbb{R}^{n_{x}}$. Moreover, it is assumed that the algebraic variables at the $n_{g}$ buses are unknown-but-bounded, that is, their values are known to lie within some confidence intervals around some nominal values. These basic assumptions allow one to reformulate (7) into the following compositional model

$$
\begin{aligned}
\dot{x}^{(1)}(t) & =\Psi^{(1)}\left(x^{(1)}(t), y^{(1)}(t)\right), y^{(1)}(t) \in \mathcal{Y}^{(1)} \\
& \vdots \\
\dot{x}^{\left(n_{g}\right)}(t) & =\Psi^{\left(n_{g}\right)}\left(x^{\left(n_{g}\right)}(t), y^{\left(n_{g}\right)}(t)\right), y^{\left(n_{g}\right)}(t) \in \mathcal{Y}^{\left(n_{g}\right)}
\end{aligned}
$$

with $\Psi^{(i)}: \mathbb{R}^{n_{x}+n_{y}} \rightarrow \mathbb{R}^{n_{x}}$ and $\mathcal{Y}^{(i)} \subset R^{n_{y}}$ as the set bounding the uncertainty of the algebraic constraints. An illustration of the proposed compositional approach is shown in Fig. 2. 
Remark 2. Here it should be stressed that (10) is a reformulation rather than being considered as a simplification of the original DAE system (7). While, this formalization may not seem substantial at first, it has several advantages:

1) The computation of the state variables for each subsystem $i$ can be parallelized.

2) The verification of each subsystem is evaluated separately, which is a much easier task compared to the verification of the complete power system.

3) Most importantly, the correlation between all machines connected to the grid is still preserved. This is due to the fact that the network interactions are included within the set of uncertainty arising from the algebraic constraints associated with the $n_{g}$ generator buses.

\section{B. Abstraction to Linear Differential Inclusions}

Our reachability algorithm is based on abstracting the differential equations of the compositional model (10) into linear differential inclusions for each consecutive time interval $\tau_{k}:=\left[t_{k}, t_{k+1}\right]$. After introducing the vector $z^{(i)}:=\left[x^{(i)^{T}} y^{(i)^{T}}\right]^{T} \in \mathbb{R}^{n_{z}}$ and the linearization point $z_{k}^{(i)}:=\left[x_{k}^{(i)^{T}} y_{k}^{(i)^{T}}\right]^{T}$, one can express the inclusion of the $i$-th generator using a first order Taylor expansion with the Lagrangian remainder

$$
\begin{aligned}
\forall t & \in\left[t_{k}, t_{k+1}\right]: \\
\dot{x}^{(i)}(t) & \in \underbrace{\left.\sum_{j=1}^{n_{x}} \frac{\partial \Psi^{(i)}\left(z^{(i)}\right)}{\partial x_{j}^{(i)}}\right|_{z^{(i)}=z_{k}^{(i)}} \Delta x_{j}^{(i)}}_{=: A_{k}^{(i)} \Delta x^{(i)}} \oplus \mathcal{U}^{(i)}\left(\tau_{k}\right)
\end{aligned}
$$

with $\Delta x^{(i)}:=x^{(i)}-x_{k}^{(i)}$ and $t_{k}:=k \cdot r$ such that $k \in \mathbb{N}$, and $r \in \mathbb{R}^{+}$corresponds to the time step and the time increment, respectively. Here $A_{k}^{(i)} \in \mathbb{R}^{n_{x} \times n_{x}}$ is the system matrix of the $i$-th machine at the time step $k$, and $\mathcal{U}^{(i)}$ is the set of uncertain inputs expressed as

$$
\begin{gathered}
\mathcal{U}^{(i)}\left(\tau_{k}\right)=\left\{u^{(i)} \in \mathbb{R}^{n_{x}}: u_{p}^{(i)}=\Psi_{p}^{(i)}\left(z_{k}^{(i)}\right) \oplus \mathcal{L}_{p}^{(i)}\left(\tau_{k}\right)\right. \\
\left.\left.\oplus \sum_{j=1}^{n_{y}} \frac{\partial \Psi_{p}^{(i)}(z)}{\partial y_{j}^{(i)}}\right|_{z=z_{k}^{(i)}}\left(y_{j}^{(i)}-y_{j, k}^{(i)}\right), y_{k}^{(i)} \in \mathcal{Y}^{(i)}\right\},
\end{gathered}
$$

with $\mathcal{L}^{(i)}\left(\tau_{k}\right)$ as the set of possible linearization errors (Lagrangian remainder) expressed in an over-approximated manner within $\tau_{k}$ according to [32]:

$$
\begin{gathered}
\mathcal{L}^{(i)}=\left\{L^{(i)} \in \mathbb{R}^{n_{x}}: L_{p}^{(i)}=\frac{1}{2} \Delta z^{(i)^{T}} H^{(i),(p)}(\zeta) \Delta z^{(i)},\right. \\
\left.z^{(i)} \in \mathcal{R}^{(i)}\left(\tau_{k}\right), \zeta \in \mathbf{I H}\left(\mathcal{R}^{(i)}\left(\tau_{k}\right)\right)\right\},
\end{gathered}
$$

with $\Delta z^{(i)}:=z^{(i)}-z_{k}^{(i)}$ and $\mathbf{I H}(\cdot)$ returning the interval hull of $\mathcal{R}\left(\tau_{k}\right)$, which denotes the reachable set within the time interval $\tau_{k}$. Here $H^{(i),(p)}, p \in\left\{1 \ldots n_{x}\right\}$ is the set of Hessian matrices, corresponding to the second-order partial derivatives of the function $\Psi^{(i)}(\cdot)$

$$
H^{(i),(p)}:=\left[\begin{array}{ccc}
\frac{\partial^{2} \Psi_{p}^{(i)}\left(z^{(i)}\right)}{\partial z_{1}^{2}} & \ldots & \frac{\partial^{2} \Psi_{p}^{(i)}\left(z^{(i)}\right)}{\partial z_{1} \partial z_{n_{z}}} \\
\vdots & \ddots & \vdots \\
\frac{\partial^{2} \Psi_{p}^{(i)}\left(z^{(i)}\right)}{\partial z_{n_{z}} \partial z_{1}} & \ldots & \frac{\partial^{2} \Psi_{p}^{(i)}\left(z^{(i)}\right)}{\partial z_{n_{z}}^{2}}
\end{array}\right],
$$

with the subscript $p$ corresponding to the $p$-th coordinate.

Remark 3. Here it should be noted that the system is continuously abstracted around a nominal value determined along the trajectory enclosed by the reachable set. Furthermore, the linear differential inclusion (11) encloses all possible nonlinear trajectories of the compositional model, since we include the set of Lagrangian remainders, which considers all linearization errors which can take place due to the linearization of (10) within $\tau_{k}$ in an over-approximative way.

\section{Computation of Over-Approximative Reachable Sets}

After defining $r:=t_{k+1}-t_{k}$ and $u_{c}^{(i)}$ as the center of $\mathcal{U}^{(i)}$, the reachable set of the dynamics $\dot{x}^{(i)}=A_{k}^{(i)} \Delta x+u_{c}^{(i)}$, enclosed by the differential inclusion (11), is based on the well-known solution of linear state-space equations

$$
\mathcal{R}_{a}^{(i)}\left(t_{k+1}\right)=e^{A_{k}^{(i)} r} \mathcal{R}^{(i)}\left(t_{k}\right) \oplus \mathcal{R}_{p}^{(i)}(r),
$$

where $e^{A_{k}^{(i)} r}$ is the matrix exponential, and $\mathcal{R}_{p}^{(i)}(r)$ is the set that over-approximates the particular solution of the linear state-space equation, according to [3, Theorem 3.1]. The reachable sets at the next point in time $t_{k+1}$ and for the time interval $\tau_{k}=\left[t_{k}, t_{k+1}\right]$ are evaluated as follows

$$
\begin{aligned}
\mathcal{R}^{(i)}\left(t_{k+1}\right) & =\mathcal{R}_{a}^{(i)}\left(t_{k+1}\right) \oplus \mathcal{R}_{u}^{(i)}(r), \\
\mathcal{R}^{(i)}\left(\tau_{k}\right) & =\mathbf{C H}\left(\mathcal{R}^{(i)}\left(t_{k}\right), \mathcal{R}_{a}^{(i)}\left(t_{k+1}\right)\right) \oplus \mathcal{R}_{e}^{(i)} \oplus \mathcal{R}_{u}^{(i)}(r),
\end{aligned}
$$

where the operator $\mathbf{C H}(\cdot)$ returns the convex hull as in (5), and the set $\mathcal{R}_{e}^{(i)}$ considers enlargement of the convex hull enclosure, in order to account for the assumption that trajectories over $\tau_{k}$ are straight lines. Finally, the reachable set $\mathcal{R}_{u}^{(i)}(r)$ accounts for the uncertainty of the input set $\mathcal{U}^{(i)}$. The evaluation of the following sets: $\mathcal{L}^{(i)}, \mathcal{R}_{p}^{(i)}(r), \mathcal{R}_{e}^{(i)}$, and $\mathcal{R}_{u}^{(i)}(r)$ are derived in our previous work [3, Ch. 3].

\section{Estimating the Set of Uncertain Inputs}

So far we have presented the computation of the reachable set of the $i$-th synchronous machine under the assumption that the set of uncertain inputs is known in advance. However, this is not the case due to the mutual dependence between the algebraic and differential variable as illustrated in Fig. 2.

First, we denote $y^{*}$ as the roots of the nonlinear algebraic equations $0=g(x, y)$ at the time step $k$. The solution may be evaluated using a variety of techniques, e.g. NewtonRaphson's method. Then, based on $y_{k}^{*}$ we make an initial guess that bounds the algebraic constraints associated with each subsystem $i$ using a multidimensional interval

$$
\begin{aligned}
\overline{\mathcal{Y}}^{(i)} & =\left[\underline{y}^{(i)}, \bar{y}^{(i)}\right], \\
\text { with } \quad \underline{y}^{(i)} & =y_{k}^{(i), *}-\gamma^{(i)}, \quad \bar{y}^{(i)}=y_{k}^{(i), *}+\gamma^{(i)},
\end{aligned}
$$


where $\gamma$ is a user-defined factor. With the knowledge of the uncertain input set, one can compute the reachable set

$$
\mathcal{R}\left(\tau_{k}\right):=\mathcal{R}^{(1)}\left(\tau_{k}\right) \times \cdots \times \mathcal{R}^{\left(n_{g}\right)}\left(\tau_{k}\right),
$$

where the sets $\mathcal{R}^{(i)}\left(\tau_{k}\right), i \in\left\{1 \ldots n_{g}\right\}$ were evaluated as described earlier in Section III-B.

Then, similar to (11), after introducing the vectors $z:=$

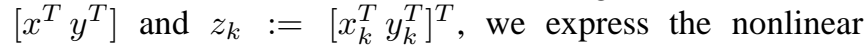
equations of the algebraic variables using a first order Taylor expansion

$$
\begin{aligned}
0= & \underbrace{\left.\sum_{j=1}^{n} \frac{\partial g(z)}{\partial x_{j}}\right|_{z=z_{k}} x_{j}}_{=: J_{k} x}+\underbrace{\left.\sum_{j=1}^{m} \frac{\partial g(z)}{\partial y_{j}}\right|_{z=z_{k}} y_{j}}_{=: W_{k} y} \\
& +\underbrace{g\left(z_{k}\right)-J_{k} x_{k}-W_{k} y_{k}}_{=: y_{0}},
\end{aligned}
$$

where $J_{k} \in \mathbb{R}^{m \times n}$ and $W_{k} \in \mathbb{R}^{m \times m}$ are the matrices of the linearized function $0=g(x, y)$. Note that the matrix $W_{k}$ is always invertible due to the index-1 property of (7), thus (19) can be reformulated as

$$
y=-W_{k}^{-1}\left(y_{0}+J_{k} x_{k}\right) .
$$

Finally by replacing the state variables by their corresponding reachable set $\mathcal{R}\left(\tau_{k}\right)$ within the time interval $\tau_{k}$, one can estimate the set of algebraic constraints using

$$
\mathcal{Y}\left(\tau_{k}\right)=\left\{y \in \mathbb{R}^{m}: y=-W_{k}^{-1}\left(y_{0} \oplus J_{k} \cdot \mathcal{R}\left(\tau_{k}\right)\right)\right\}
$$

which involves a linear transformation of the set of dynamic state variables. In the event that $\exists i: \mathcal{Y}^{(i)}\left(\tau_{k}\right) \nsubseteq \overline{\mathcal{Y}}^{(i)}$, one must further enlarge the initial guess $\overline{\mathcal{Y}}^{(i)}, \forall i \in\left\{1, \ldots, n_{g}\right\}$ and recompute the reachable set.

\section{E. Overall Algorithm}

The complete procedure to compute reachable sets of power systems modelled by (7), using the proposed compositional approach, is outlined in Alg. 1. First, the Cartesian product of the initial sets for each synchronous generator is computed and the set of uncertainty due to the algebraic constraints is estimated. Then, the algorithm examines two loops: the first loop computes the reachable set for the specified fault scenario and determines if the reachable set converges back to the equilibrium/stability region. The second loop parallelizes the computation of the reachable sets of the synchronous machines at each time step $\tau_{k}$ and verifies if the set of algebraic constraints at each $n_{g}$ bus is enclosed by the initial guess.

\section{RESUlts}

This section illustrates the application of our proposed compositional algorithm on three benchmark examples. All computations are performed in MATLAB2014b on a standard computer. Our algorithm computes forward reachable sets using the CORA toolbox [33]. We compare the computational time with the algorithm described in our previous contribution [14].

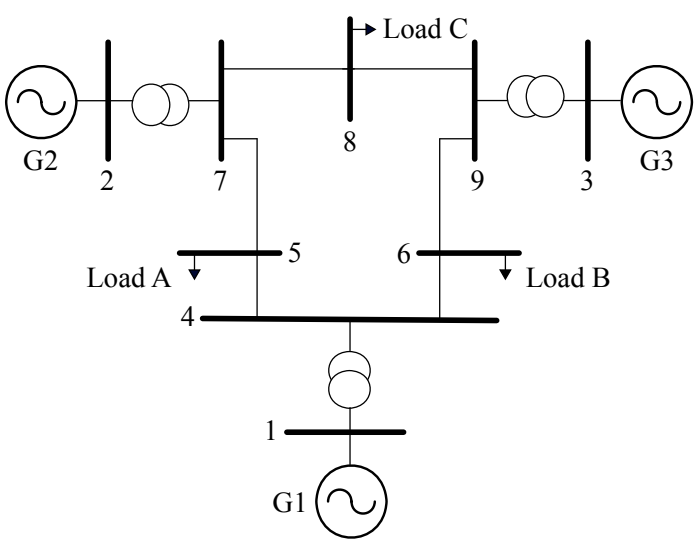

Fig. 3. The 3-machine 9-bus benchmark [18, Ch. 2].

\section{A. Modelling of Power Systems}

In this work, the dynamics synchronous machines are expressed by the swing equation

$$
\begin{aligned}
& \dot{\delta}_{i}=\omega_{i}-\omega_{r}, \\
& \dot{\omega}_{i}=1 / M\left(P_{i}^{m}-P_{i}^{g}-D_{i}\left(\omega_{i}-\omega_{r}\right)\right),
\end{aligned}
$$

with: $P_{i}^{g}=E_{i} V_{i}\left|Y_{i}^{g}\right| \cos \left(\Theta_{i}^{g}+\delta_{i}-\theta_{i}\right)-V_{i}^{2}\left|Y_{i}^{g}\right| \cos \left(\Theta_{i}^{g}\right)$,

where $\delta$ is the rotor angle and $\omega$ is the angular velocity. The subscript $i$ represents the $i$-th generator. It is assumed that the mechanical power $P^{m}$ and the generator voltage $E$ are kept constant, which can be justified for studies involving transient stability analysis [17]. The constants $M, D, \omega_{r}$, $Y^{g}$, and $\Theta^{g}$ correspond to the rotational inertia, the damping of the generator shaft, the reference synchronous speed, the absolute value, and the phase angle of the admittance from the generator to its connected bus, respectively.

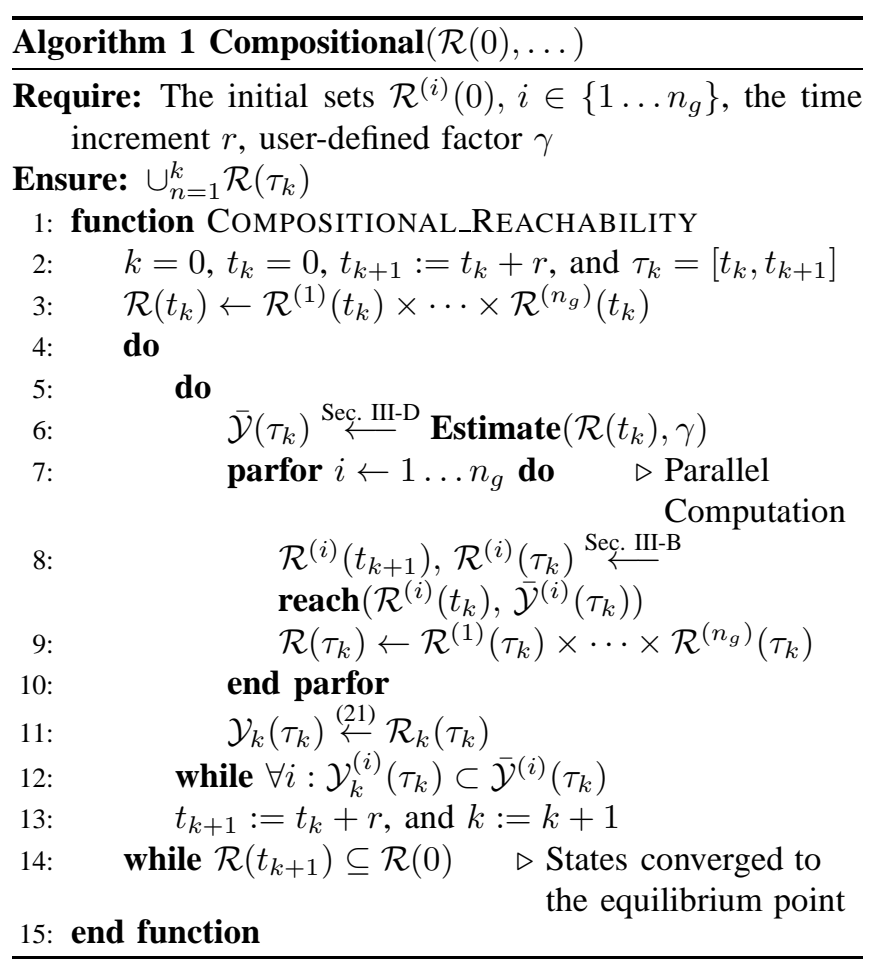


TABLE I

COMPARISON OF THE CPU TIME FOR DIFFERENT BENCHMARK EXAMPLES.

\begin{tabular}{ccccc}
\hline \multirow{2}{*}{ Benchmark example } & \multicolumn{2}{c}{ Computational time } & \multicolumn{2}{c}{ State variables } \\
& Proposed algorithm & Algorithm in [14] & Dynamic & Algebraic \\
\hline 1-machine 2-bus [17, Ch. 12] & $9.78 \mathrm{~s}$ & $10.14 \mathrm{~s}$ & 2 & 4 \\
2-machine 3-bus [34, Example 3] & $24.2 \mathrm{~s}$ & $51.73 \mathrm{~s}$ & 4 & 8 \\
3-machine 9-bus [18, Ch. 2] & $50.72 \mathrm{~s}$ & $20 \mathrm{~min}$ & 6 & 35 \\
\hline
\end{tabular}

The constraints $V$ and $\theta$, denoting the bus voltage and phase angle, respectively, are associated with the grid algebraic equations. They are calculated using the power flow equations

$$
\begin{aligned}
P_{j} & =P_{j}^{g}-P_{j}^{d}, \\
& =V_{j} \sum_{k=1}^{b} V_{j}\left|Y_{j k}\right| \cos \left(\Theta_{j k}-\theta_{k}-\theta_{j}\right), \\
Q_{j} & =Q_{j}^{g}-Q_{j}^{d}, \\
& =-V_{j} \sum_{k=1}^{b} V_{j}\left|Y_{j k}\right| \sin \left(\Theta_{j k}-\theta_{k}-\theta_{j}\right),
\end{aligned}
$$

where $P$ and $Q$ denote the active and reactive power injections at the $j$-th bus, respectively. The superscript $d$ denotes the power demand from constant loads. Finally, the line admittance is expressed by the absolute value $Y$ and the phase angle $\Theta$.

\section{B. Examples}

We consider three common benchmark examples to showcase the applicability and scalability of our proposed algorithm. The first system is the so-called SMIB system [17, Ch. 12] consisting of a synchronous generator connected to

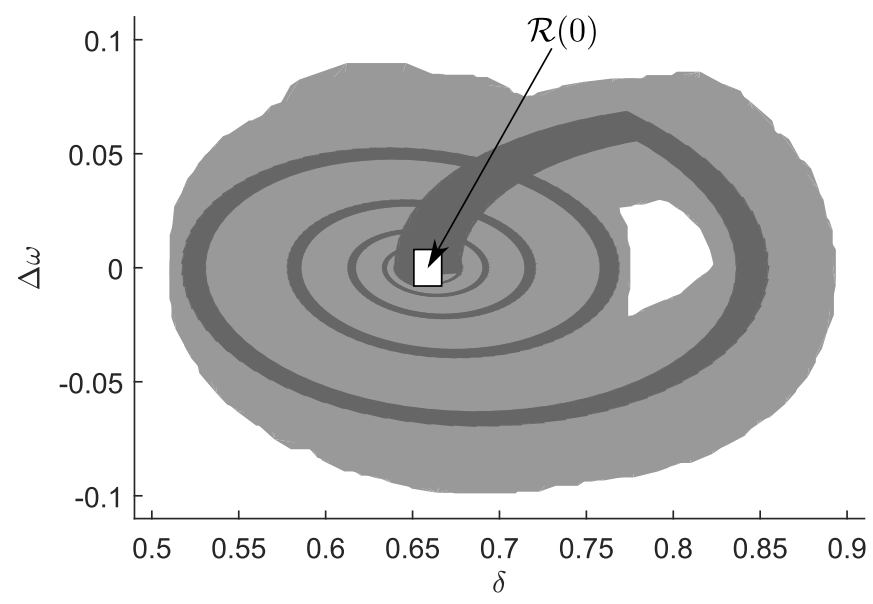

Fig. 4. Projection of the dynamic state variable of the SMIB system. The projections show a comparison between the reachable set using the proposed compositional technique (gray area), outlined in Alg. 1, and those computed using our algorithm presented in [14] (dark gray area). The initial set of the generator dynamic state variables $\mathcal{R}(0)$ is the white box. The computation of the reachable sets are performed until $\mathcal{R}\left(t_{k}\right) \subseteq \mathcal{R}(0)$ to prove that all states were attracted by the stability region. an infinite bus whose voltage and phase angle are known and kept constant. The SMIB system consists of six state variables: two variables correspond to the generator dynamic states appearing in (22) and four algebraic variables associated with the constraints at the generator bus.

The second system is the DMIB system [34, Example 3], which is slightly larger than the SMIB system. This system consists of two synchronous machines connected to an infinite bus via a three bus transmission network resulting in a total of 12 state variables.

The final system to consider is the WSCC 9-bus test case as illustrated in Fig. 3. The system represents a simple approximation of the Western System Coordinating Council
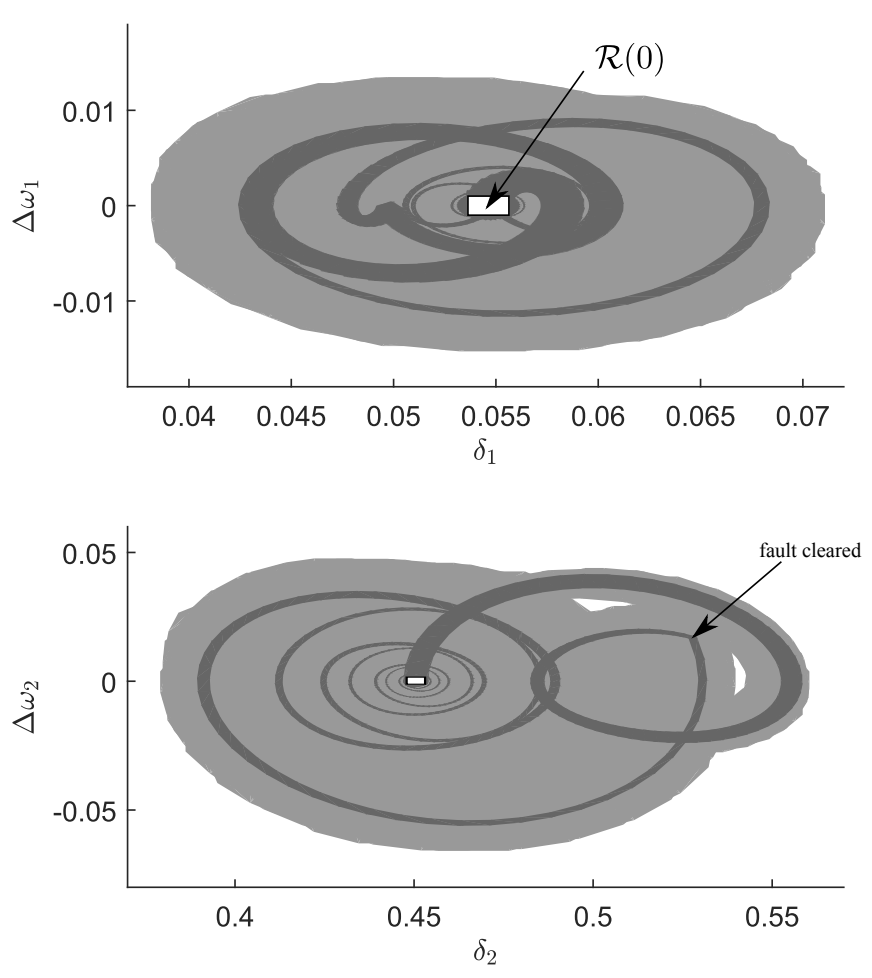

Fig. 5. Chosen projections of the dynamic state variables of the WCSS 9-bus benchmark. The projections show a comparison between the reachable set using the proposed compositional technique (gray area), outlined in Alg. 1, and those computed using our algorithm presented in [14] (dark gray). The initial set of the generator dynamic state variables $\mathcal{R}(0)$ is the white box. The considered fault scenario is the loss of the transmission line connecting the buses 5 and 7 . The line is reconnected after the clearance at the fault, and the reachable set is computed until all states are enclosed by $\mathcal{R}(0)$. 

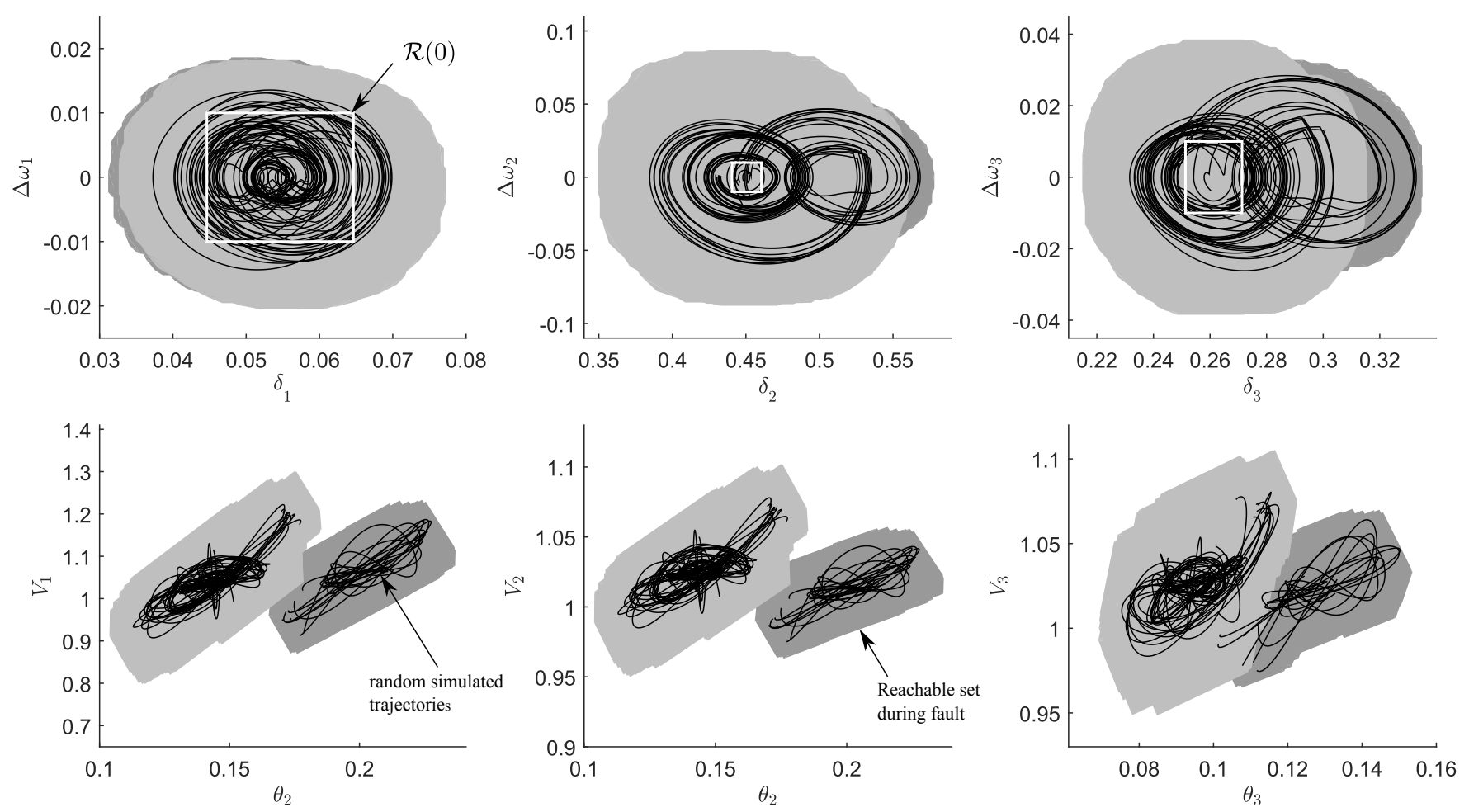

Fig. 6. Projection of selected differential and algebraic variables for the WCSS 9-bus power system. The dark gray areas show the reachable set during faults. The considered fault scenario is the loss of the transmission line connecting the buses 5 and 7 . The line is reconnected after the clearance at the fault, and the reachable set is computed until all states are enclosed by $\mathcal{R}(0)$. The solid lines present random simulation results starting from the edges of the initial reachable set $\mathcal{R}(0)$.

(WSCC) to an equivalent system with 9 buses and 3 generators [18, Ch. 2] with a total of 41 state variables.

The fault scenario we consider for the benchmark examples is the loss of one transmission line, followed by its reconnection to the network following the clearance of the fault. The PQ-loads of the 9-bus power systems are modelled as constant impedances and in transient response, their dynamics are modelled by constant admittances [30, p. 258].

\section{Computation of Reachable Sets}

Fig. 4-6 illustrate the computed reachable set according to the proposed algorithm outlined in Alg. 1. To validate our results, we compare the resulting reachable sets to those obtained when considering the full system, i.e., no compositional techniques are applied. The reachability algorithm used in the comparison is described in detail in our previous contribution [14] and considers power systems modelled by the original set of nonlinear DAEs (7). Moreover, we simulate the DAE system (7) using the ODE-15s to verify and ensure if the nonlinear trajectories are enclosed by the computed reachable set. The computation of the reachable sets is performed until all states are enclosed by the initial set to guarantee that all state variables converged to the equilibrium point.

It can be seen that our proposed compositional algorithm provides fairly accurate results compared to the reachable sets computed for the exact DAE system (7). Furthermore, the nonlinear trajectories of the dynamic and algebraic variables of the simulated DAE system are enclosed by the reachable sets. The computational times for the three benchmark examples are listed in Table I. It is obvious that the computational resources are drastically reduced when computing the reachable set in a compositional way for the 3-machine 9-bus system; however, the CPU time when computing the set of the SMIB system is comparable, due to the simplicity of the system. The computational time for the DMIB using the exact algorithm is still feasible even though it is clearly outperformed by the new algorithm.

Our proposed algorithm, however, introduces some conservatism, which can be considered a tradeoff between accuracy and efficiency. This conservatism results from the uncertainty of the input set associated with the algebraic constraints at the $n_{g}$ generator buses. This leads to further over-approximation of the reachable set since we consider all possible values taken by the bus voltage and phase angle, even the unrealistic ones. It should be noted that the conservatism does not affect the security assessment during transient response; however, it can degrade the performance of the system, if the computed reachable set intersects with safety limits, e.g., bus voltage exceeding limits defined by the grid operators. Reducing and even eliminating the resulting conservatism will be further investigated in future work.

\section{CONCLUSion}

We presented a new algorithm for compositional transient stability analysis of power systems via the computation of 
reachable sets. Using our proposed technique, we drastically reduced the computational time required to compute the reachable set compared to our previous contribution [14]. The main reason for the improved algorithmic efficiency is that we reformulate the complete power system into a set of subsystems, each consisting of a synchronous machine connected to a generator bus, whose algebraic constraints are unknown-but-bounded within some confidence intervals. This makes it possible to parallelize the computation of the reachable set for each synchronous generator, while, most importantly, preserving the interaction between different machines connected to the grid during faults. The applicability of the algorithm has been illustrated on various benchmark examples, and the tradeoff between accuracy and algorithmic efficiency is demonstrated by validating our results against the results of our previous work [14].

\section{ACKNOWLEDGMENT}

Financial support by the German Research Foundation (DFG) is gratefully acknowledged (AL 1185/2-1).

\section{REFERENCES}

[1] I. M. Mitchell, "Comparing forward and backward reachability as tools for safety analysis," in Hybrid systems: computation and control. Springer, 2007, pp. 428-443.

[2] C. Le Guernic, "Reachability analysis of hybrid systems with linear continuous dynamics," Ph.D. dissertation, Université Joseph-FourierGrenoble I, 2009.

[3] M. Althoff, "Reachability analysis and its application to the safety assessment of autonomous cars," Ph.D. dissertation, Technische Universität München, 2010.

[4] P. Mohajerin Esfahani, M. Vrakopoulou, K. Margellos, J. Lygeros, and G. Andersson, "Cyber attack in a two-area power system: Impact identification using reachability," in Proc. of the IEEE American Control Conference, 2010, pp. 962-967.

[5] M. Vrakopoulou, P. M. Esfahani, K. Margellos, J. Lygeros, and G. Andersson, "Cyber-attacks in the automatic generation control," in Cyber Physical Systems Approach to Smart Electric Power Grid. Springer, 2015, pp. 303-328.

[6] A. El-Guindy, D. Han, and M. Althoff, "Formal analysis of drumboiler units to maximize the load-following capabilities of power plants," IEEE Transactions on Power Systems, vol. 31, no. 6, pp. 46914702, 2016.

[7] V. Pico, N. Hugo, and D. C. Aliprantis, "Voltage ride-through capability verification of wind turbines with fully-rated converters using reachability analysis," IEEE Transactions on Energy Conversion, vol. 29, no. 2, pp. 392-405, 2014.

[8] Y. C. Chen and A. D. Domínguez-García, "A method to study the effect of renewable resource variability on power system dynamics," IEEE Transactions on Power Systems, vol. 27, no. 4, pp. 1978-1989, 2012.

[9] X. Jiang, Y. C. Chen, and A. D. Dominguez-Garcia, "A set-theoretic framework to assess the impact of variable generation on the power flow," IEEE Transactions on Power Systems, vol. 28, no. 2, pp. 855867, 2013.

[10] M. Kamgarpour, C. Beyss, and A. Fuchs, "Reachability-based control synthesis for power system stability," IFAC-PapersOnLine, vol. 49, no. 27, pp. 238-243, 2016.

[11] A. El-Guindy, K. Schaab, B. Schürmann, O. Stursberg, and M. Althoff, "Formal LPV control for transient stability of power systems," in Proc. of the 2017 IEEE Power and Energy Society General Meeting, 2017, pp. 1-5.

[12] L. Jin, R. Kumar, and N. Elia, "Reachability analysis based transient stability design in power systems," International Journal of Electrical Power \& Energy Systems, vol. 32, no. 7, pp. 782-787, 2010.

[13] A. El-Guindy, D. Han, and M. Althoff, "Estimating the region of attraction via forward reachable sets," in Proc. of the IEEE American Control Conference, 2017.
[14] M. Althoff and B. H. Krogh, "Reachability analysis of nonlinear differential-algebraic systems," IEEE Transactions on Automatic Control, vol. 59, no. 2, pp. 371-383, 2014.

[15] C. P. Steinmetz, "Power control and stability of electric generating stations," Transactions of the American Institute of Electrical Engineers, vol. 39 , no. 2, pp. 1215-1287, 1920.

[16] P. Kundur, J. Paserba, V. Ajjarapu, G. Andersson, A. Bose, C. Canizares, N. Hatziargyriou, D. Hill, A. Stankovic, and C. Taylor, "Definition and classification of power system stability IEEE/CIGRE joint task force on stability terms and definitions," IEEE Transactions on Power Systems, vol. 19, no. 3, pp. 1387-1401, 2004.

[17] P. Kundur, N. J. Balu, and M. G. Lauby, Power system stability and control. McGraw-hill New York, 1994, vol. 7.

[18] P. M. Anderson and A. A. Fouad, Power System Control and Stability. Wiley-IEEE Press, 2002.

[19] L. Jin, H. Liu, R. Kumar, J. D. Mc Calley, N. Elia, and V. Ajjarapu, "Power system transient stability design using reachability based stability-region computation," in Proceedings of the 37th Annual North American Power Symposium. IEEE, 2005, pp. 338-343.

[20] E. A. Cross and I. M. Mitchell, "Level set methods for computing reachable sets of systems with differential algebraic equation dynamics," in Proc. of the IEEE American Control Conference, 2008, pp. 2260-2265.

[21] I. M. Mitchell, A. M. Bayen, and C. J. Tomlin, "A time-dependent Hamilton-Jacobi formulation of reachable sets for continuous dynamic games," IEEE Transactions on Automatic Control, vol. 50, no. 7, pp. 947-957, 2005.

[22] Y. Susuki, T. Sakiyama, T. Ochi, T. Uemura, and T. Hikihara, "Verifying fault release control of power system via hybrid system reachability," in Proc. of the 40th North American on Power Symposium, 2008, pp. 1-6.

[23] Y. Susuki, T. J. Koo, H. Ebina, T. Yamazaki, T. Ochi, T. Uemura, and T. Hikihara, "A hybrid system approach to the analysis and design of power grid dynamic performance," Proc. of the IEEE, vol. 100, no. 1, pp. 225-239, 2012.

[24] A. Girard, "Reachability of uncertain linear systems using zonotopes," in Hybrid Systems: Computation and Control. Springer, 2005, pp. 291-305.

[25] M. Althoff, O. Stursberg, and M. Buss, "Reachability analysis of nonlinear systems with uncertain parameters using conservative linearization," in Proc. of the 47th IEEE Conference on Decision and Control, 2008, pp. 4042-4048.

[26] X. Chen, E. Ábrahám, and S. Sankaranarayanan, "Flow*: An analyzer for non-linear hybrid systems," in International Conference on Computer Aided Verification, 2013, pp. 258-263.

[27] T. Dang, A. Donzé, and O. Maler, "Verification of analog and mixed-signal circuits using hybrid system techniques," in International Conference on Formal Methods in Computer-Aided Design. Springer, 2004, pp. 21-36.

[28] Z. Han and B. H. Krogh, "Reachability analysis of large-scale affine systems using low-dimensional polytopes," in International Workshop on Hybrid Systems: Computation and Control. Springer, 2006, pp. 287-301.

[29] A. B. Kurzhanski and P. Varaiya, "Ellipsoidal techniques for reachability analysis: internal approximation," Systems \& control letters, vol. 41, no. 3, pp. 201-211, 2000.

[30] F. Milano, Power system modelling and scripting. Springer Science \& Business Media, 2010

[31] A. Platzer and E. M. Clarke, "The image computation problem in hybrid systems model checking," in Hybrid Systems: Computation and Control. Springer, 2007, pp. 473-486.

[32] M. Berz and G. Hoffstätter, "Computation and application of Taylor polynomials with interval remainder bounds," Reliable Computing, vol. 4, no. 1, pp. 83-97, 1998.

[33] M. Althoff, "An Introduction to CORA 2015," in Proc. of the Workshop on Applied Verification for Continuous and Hybrid Systems, 2015, pp. 120-151, http://www6.in.tum.de/Main/SoftwareCORA.

[34] N. G. Bretas and L. F. Alberto, "Lyapunov function for power systems with transfer conductances: Extension of the invariance principle," IEEE Transactions on Power Systems, vol. 18, no. 2, pp. 769-777, 2003. 